\title{
Tiempos de pandemia y enseñanza universitaria: recrear las propuestas metodológicas entre el hogar y las plataformas de medios conectivos
}

\author{
Times of Pandemic and Higher Education Teaching: reinventing \\ methodological proposals between home and connective media platforms
}

\section{Tempos de pandemia e ensino universitário: recriar as propostas metodológicas entre a casa e a as plataformas de mídia conectiva}

\author{
Ana Griselda Diaz \\ Universidad Nacional de Catamarca \\ Catamarca, Argentina \\ anagriseldadiaz@gmail.com \\ (1D) https:///orcid.org/0000-0003-0602-1868
}

Recibido - Received - Recebido: 23 / 07 / 2020 Corregido - Revised - Revisado: 17 / 08 / 2020 Aceptado - Accepted - Aprovado: 24 / 09 / 2020

DOl: https://doi.org/10.22458/ie.v22iespecial.3216

URL: https://revistas.uned.ac.cr/index.php/innovaciones/article/view/3216

\begin{abstract}
Resumen: Las innovaciones comunicacionales conllevan cambios en los modos de interacción y desarrollo social. Para afrontarlos, se demanda a las universidades la inclusión de nuevos contenidos y formas de enseñanza que posibiliten el desempeño de los futuros profesionales en escenarios cambiantes e inciertos. En Argentina, la pandemia desestabilizó la forma más clásica de mediación pedagógica en el nivel superior: la explicación del docente en vivo, simultánea y en espacios físicos cerrados.

La emergente virtualización de carreras universitarias presenciales expuso que los tradicionales modos de comunicación del conocimiento solo eran compatibles con un sistema único de realidad analógico y tangible. Los medios conectivos que hoy posibilitan la continuidad pedagógica de los trayectos de formación profesional requieren el aprendizaje de habilidades tecnológicas tanto de los formadores para la enseñanza de los contenidos como de los estudiantes para la apropiación de estos. Los objetivos del presente ensayo son: a) replantear los actuales procesos de mediación pedagógica y tecnológica de las prácticas de la enseñanza universitaria cuyas aulas y clases se despliegan entre el ecosistema de medios conectivos y los hogares de docentes y estudiantes, b) exponer conceptualmente la relevancia de la categoría construcción metodológica como posibilidad para comprender y recrear los modos de intervención didáctica en el nivel superior universitario. Desde los enfoques de la educación inclusiva, las Pedagogías mediáticas y las perspectivas contemporáneas de la Didáctica se sostiene que en las construcciones metodológicas se revelan innovaciones didácticas que habilitan procesos de mediación y apropiación tecnológica en escenarios contemporáneos desiguales y atravesados por ecosistemas tecnológicos.
\end{abstract}

Palabras clave: Enseñanza superior, Enseñanza multimedia, Medios sociales, Pandemia, COVID-19

\begin{abstract}
Innovations in communications entail changes in the modes of interaction and social development. In order to face those changes, Universities are required to incorporate new contents as well as teaching techniques that enable future professionals to perform in changing and uncertain scenarios. In Argentina, the pandemic destabilized higher education's most prevailing type of pedagogical mediation: live, simultaneous explanations in enclosed physical spaces. The emerging virtualization of face-to-face university courses has revealed that the traditional modes of knowledge communication were only compatible with a single system of analog and tangible reality. Connective media enables the pedagogical continuity of vocational training nowadays and it requires the learning of technological skills from both the teachers, so they can teach the contents, and from the students for knowledge appropriation. The objectives of this essay are: a) To rethink the current pedagogical
\end{abstract}


and technological mediation processes of university teaching practices where classrooms and classes unfold between the ecosystem of connective media and the homes of teachers and students. b) To conceptually bring to light the relevance of the methodological construction category as a possibility to understand and recreate the modes of didactic intervention at a higher education level. Considering approaches to inclusive education, media-based learning, and contemporary didactics perspectives, it is argued that, in the methodological constructions didactic innovations are revealed to be enablers of mediation processes and technological appropriation in unequal contemporary scenarios overpassed by technological ecosystems.

Key Words: Higher Education, Multimedia Teaching, Social Media, Pandemic, COVID-19

Resumo: As inovações comunicacionais levam a mudanças nos modos de interação e desenvolvimento social. Para enfrentá-las, as universidades devem incluir novos conteúdos e formas de ensino que permitam o desempenho dos futuros profissionais em cenários de mudança e incertezas. Na Argentina, a pandemia desestabilizou a forma mais clássica de mediação pedagógica no nível superior: a explicação do professor ao vivo, simultaneamente e em espaços físicos fechados.

A emergente virtualização de carreiras universitárias presenciais evidenciou que os modos tradicionais de comunicação do conhecimento só eram compatíveis com um sistema único de realidade analógica e tangível. Os meios de comunicação conectivos possibilitam hoje a continuidade pedagógica dos percursos de formação profissional exigem a aprendizagem de habilidades tecnológicas tanto dos formadores para o ensino dos conteúdos quanto dos estudantes para a apropriação destes. Os objetivos do presente ensaio são: a) repensar os atuais processos de mediação pedagógica e tecnológica das práticas do ensino universitário cujas salas de aulas se desdobram entre o ecossistema de meios de conexão e casas de professores e estudantes, b) expor conceitualmente a relevância da categoria construção metodológica como uma possibilidade para compreender e recriar os modos de intervenção didática no nível superior universitário. A partir de enfoques da educação inclusiva, as Pedagogias mediáticas e perspectivas contemporâneas da Didática argumenta-se que as construções metodológicas se relevam inovações didáticas que habilitam processos de mediação e apropriação tecnológica em cenários contemporâneos desiguais e atravessados por ecossistemas tecnológicos.

Palavras-chave: Ensino superior, ensino multimídia, mídia social. Pandemia, COVID-19

\section{INTRODUCCIÓN}

Actualmente la enseñanza universitaria se desarrolla en escenarios múltiples y complejos habitados por prácticas que oscilan entre la tradición y la búsqueda de transformación. Desde mediados del siglo pasado, y con mayores implicancias institucionales en el siglo XXI, las Tecnologías de la Información y la Comunicación (TIC) irrumpen en la actividad de la enseñanza universitaria como un nuevo lenguaje que instaura y mediatiza modos de comunicación que perforan la identidad de los futuros profesionales (Meléndez, Diaz y Yuni, 2017).

La emergencia sanitaria, causada por la propagación del virus de la COVID-19, generó el traslado de todas las carreras de grado de la Universidad Nacional de Catamarca (UNCA) - Argentina, a una modalidad de enseñanza remota mediada por tecnologías digitales. Estas circunstancias evidenciaron problemas ya existentes y provocaron eventos imprevistos. Hoy en día pensar la formación como campo complejo (Souto, 2009) en la que coexisten el orden y el desorden, el tejido de eventos, interacciones, retroacciones, determinaciones, azares que constituyen nuestro mundo fenoménico; llega a su máxima expresión.

En palabras de Morín (2000, p. 60) "la complejidad coincide con un aspecto de incertidumbre, ya sea en los límites de nuestro entendimiento, ya sea inscrita en los fenómenos (...) es la incertidumbre en el seno de los sistemas ricamente organizados". Desde esta perspectiva, "el evento es el orden de lo improbable, lo azaroso, lo aleatorio, lo accidental, lo imprevisible, lo singular y lo concreto. (...) tiene lugar en el devenir y puede significar cambios a los que hay que estar abierto" (Souto, 2009, p. 17). Seguidamente, se destacan en orden cronológico aquellos eventos cuya imprevisibilidad, celeridad y singularidad marcaron el devenir de la educación Argentina y particularmente del nivel superior universitario en tiempos de pandemia: 
- 14 de marzo: el ministro de educación nacional mediante la resolución 104/2020 recomienda a las universidades, institutos universitarios y de educación superior de todas las jurisdicciones, que adecuen las condiciones en que se desarrolla la actividad académica presencial en el marco de la emergencia conforme con las recomendaciones del ministerio de salud procurando garantizar el desarrollo del calendario académico, los contenidos mínimos de las asignaturas y su calidad. Esto podrá contemplar la implementación transitoria de modalidades de enseñanza a través de los campus virtuales, medios de comunicación o cualquier otro entorno digital del que dispongan.

- 16 de marzo: el Rector de la UNCA ad-referéndum del Consejo Superior mediante resolución $n^{\circ} 102 / 2020$, faculta a las autoridades de las Unidades Académicas a la reprogramación de las clases presenciales a partir del 18 de marzo y, en consonancia con las recomendaciones del Ministerio de Educación Nacional, las Unidades Académicas deberán adoptar las medidas y procedimientos de enseñanza adecuados que garanticen los contenidos mínimos de las asignaturas y su calidad académica. Para ello, las clases podrán realizarse a través de los campus virtuales existentes en el Rectorado y las distintas Unidades Académicas o cualquier entorno digital disponible.

- 19 de marzo: el presidente de la Nación en acuerdo general de ministros, mediante el Decreto $n^{\circ} 297 / 2020$ dispone la medida de Aislamiento Social, Preventivo y Obligatorio (ASPO). Durante su vigencia las personas deberán permanecer en sus residencias habituales o en la residencia en que se encuentren a las 00:00 horas del día 20 de marzo de 2020, abstenerse de concurrir a sus lugares de trabajo y no podrán desplazarse por rutas, vías y espacios públicos con el fin de prevenir la circulación y el contagio del virus de la COVID-19 y la consiguiente afectación a la salud pública. Quedan exceptuadas del cumplimiento del ASPO y de la prohibición de circular, las personas afectadas a las actividades y servicios declarados esenciales en la emergencia.

Estos eventos provocaron el traslado compulsivo y forzoso de las clases y aulas universitarias a los hogares de estudiantes y docentes mediados por tecnologías digitales generando alteraciones en el orden didáctico y pedagógico. En el plano didáctico se replantearon los procesos de selección de los contenidos para decidir aquellos conocimientos mínimos, en términos de lo irrenunciable, importante y necesario que un futuro profesional debe aprender y, con estas decisiones, se evidenció la necesidad de reconstruir las formas de transmisión del contenido en estas circunstancias. Desde la dimensión pedagógica es clave repensar qué sujeto se pretende formar, por qué y para qué educar, qué habilidades, competencias, actitudes y valores deberían desarrollar los estudiantes para desempeñarse en escenarios y entornos profesionales que también fueron alterados.

Propiciar el desarrollo de capacidades y competencias con los futuros profesionales es una de las tareas específicas que asume el personal docente de nivel superior. Para lograrlo, se considera necesario investigar los procesos metodológicos que se despliegan en las prácticas de la enseñanza universitaria. En este sentido, se entiende los procesos metodológicos desde el enfoque propuesto por Gloria Edelstein (1996) quién los define como construcciones metodológicas. Las investigaciones desarrolladas por Edelstein cuestionaron los tradicionales modos de entender y significar lo metodológico en el campo de la Didáctica, asumiendo como punto clave de sus postulados la consideración del docente como sujeto creador de propuestas de intervención que se rigen por una lógica particular en su construcción. Desde esta perspectiva, la construcción metodológica no es absoluta sino relativa, ya que se conforma teniendo en cuenta la disciplina, los sujetos en situación de apropiarse de ella y los ámbitos particulares en las que se inscriben (Edelstein, 1996).

La pandemia, como situación no prevista y circunstancial, puso en jaque los posicionamientos asumidos en tiempos prepandémicos. La inclusión de las TIC y de tecnologías digitales a las prácticas de la enseñanza del nivel superior dependía de cada docente. Pero, durante el periodo de ASPO, las construcciones metodológicas que despliega el personal docente son posibles con mediaciones tecnologías en entornos virtuales. En atención a lo planteado y ante la imposibilidad de dar continuidad a los trayectos 
formativos mediante un régimen de cursado presencial, los objetivos del presente ensayo son: a) replantear los actuales procesos de mediación pedagógica y tecnológica de las prácticas de la enseñanza universitaria cuyas aulas y clases se despliegan entre el ecosistema de medios conectivos y los hogares de docentes y estudiantes, b) exponer conceptualmente la relevancia de la categoría construcción metodológica como posibilidad para comprender y recrear los modos de intervención didáctica en el nivel superior universitario.

\section{ENSEÑAR EN TIEMPOS DE PANDEMIA: UN ENCUENTRO HUMANO MEDIADO POR TECNOLOGÍAS DIGITALES EN ESCENARIOS DESIGUALES}

Referentes clave de la didáctica definen a las prácticas de la enseñanza como acciones humanas, institucionales, sociales y políticas (Camilloni, 1996, 2007; Bolivar y Bolivar Ruso, 2011; Davini, 2008; Litwin, 1996; Pruzzo, 2015; Maggio, 2012, 2016, 2018). La enseñanza implica un encuentro humano y, aunque hoy esté entramada en los denominados ecosistemas tecnológicos (Van Dijck, 2016), su eje radica en generar diálogos genuinos que posibiliten la construcción colectiva del conocimiento y no solo la transmisión lineal de información.

Porque enseñar es, en definitiva, participar en el proceso de formación de otra persona, tarea que solo puede hacerse en sentido pleno con ese otro. Los docentes (...) saben del mágico momento en el que la intención pedagógica se encuentra con el deseo, la motivación, la voluntad y el proyecto del otro. Saben también de la desazón y el malestar que genera su ausencia. Casi una afrenta a la omnipotencia pedagógica que no hace más que recordar que ese otro es un sujeto capaz de libertad que puede, incluso, resistir a los intentos de influencia que se ejerzan sobre él. (Basabe y Cols, 2007, pp. 146-147).

En este encuentro humano el profesorado media la relación entre los estudiantes y determinados saberes. Pero mediar el conocimiento no significa pasar información de un ángulo a otro del clásico del triángulo pedagógico. La mediación pedagógica implica facilitar al estudiantado el acceso y apropiación a determinados saberes y objetos culturales, genera situaciones de enseñanza que les posibiliten la construcción de renovados conocimientos. Esta mediación "ubica al docente en una condición bifronte: está de cara al alumno, pero también ocupa una particular posición en relación con el saber" (Basabe y Cols, 2007, p. 147). Siguiendo este pensamiento, no se trata solo del conocimiento que el docente tenga de su materia, el saber enseñado lleva la marca personal del proceso que dio lugar a su construcción y de los significados que el docente construye con relación a ese saber.

En tiempos de aislamientos social para reconstruir los procesos de mediación pedagógica se requieren mediaciones tecnológicas. En la UNCA cada unidad académica validó el uso de diferentes plataformas digitales y a su vez, en algunas facultades, cada docente decidió conforme a sus posibilidades y las de sus estudiantes, el medio digital para reconstruir sus clases. No obstante, si se tienen en cuenta las recomendaciones del Ministerio de Educación respecto de asegurar los contenidos mínimos y su calidad académica, algunos plantean si es posible brindar calidad académica usando cualquier entorno digital. En términos de Sibilia (2019):

(...) las tecnologías no son neutras porque son históricas y, justamente por eso, cargan consigo una serie de creencias y valores propios de su época. Todos los artefactos, (...) si bien se pueden usar para hacer muchas cosas, no es cierto que se puedan usar para hacer "cualquier cosa". Cada herramienta supone, propone y estimula ciertos modos de usarla (y no otros), que a su vez implican determinados modos de vivir (pp. 5-6). 
En tiempos de pandemia el uso combinado de diferentes aplicaciones y entornos digitales como por ejemplo WhatsApp ${ }^{\oplus}$, Facebook ${ }^{\circledast}$, Google Classroom ${ }^{\circledast}$, Moodle, Zoom ${ }^{\circledast}$, permite comunicar muchos contenidos, no obstante, el tratamiento de los saberes culturales y los aprendizajes que se pretenden lograr varían, entre otras dimensiones, según las finalidades formativas de la carrera, las funcionalidades que ofrecen las herramientas, las habilidades de los sujetos para usar las tecnologías pedagógicamente, los servicios de conectividad y dispositivos tecnológicos disponibles.

Es posible pensar que la recomendación de utilizar cualquier entorno digital para dar continuidad a las clases se vincula al diferenciado acceso y uso de las TIC en los hogares argentinos. De acuerdo con los indicadores relevados por el Instituto Nacional de Estadística y Censos (INDEC) a partir de la Encuesta Permanente de Hogares (EPH) mediante el Módulo de Acceso y Uso de las Tecnologías de la Información y la Comunicación (MAUTIC), en el cuarto trimestre de 2019, se registró que el 60,9\% de los hogares urbanos tiene acceso a computadora y el 82,9\%, a internet. Los datos muestran que, en la Argentina, 84 de cada 100 personas emplean teléfono celular y 80 de cada 100 utilizan internet. En términos comparativos, se registra un incremento en el uso de internet y de teléfono celular; acompañados de un descenso en la utilización de computadoras. El celular es la tecnología con mayor uso, debido a que 8 de cada 10 habitantes emplean teléfono móvil.

En el caso del Gran Catamarca, donde se encuentra situada la UNCA, los datos relevados a través de la EPH señalan que el $80,6 \%$ de los hogares usa internet, el $32,3 \%$ usa la computadora y el $85 \%$ usa teléfono celular. Cabe mencionar que la provincia de Catamarca corresponde a la región Noroeste de Argentina, su población es de 396.895 habitantes y se denomina Gran Catamarca a la aglomeración urbana que se extiende desde la ciudad de San Fernando del Valle de Catamarca, a los departamentos Valle Viejo y Fray Mamerto Esquiú representando la aglomeración más poblada de la provincia. Según el censo 2010 el gran Catamarca cuenta con 200.100 habitantes.

De acuerdo con los datos del MAUTIC 2019, hay más hogares que acceden a internet que a computadora. En el contexto nacional, en la región Noroeste el $86,4 \%$ de los hogares accede a internet, pero el $57,3 \%$ a una computadora. El empleo de computadora sin utilizar internet aparece más relegado en relación con el uso de las otras tecnologías relevadas. Esta información es valiosa al momento de generar las propuestas de enseñanza y aprendizaje puesto que analizar, explorar, leer grandes volúmenes de información en diferentes formatos y por tiempos prolongados requiere el uso de computadoras (móviles o de escritorio). De igual modo, según las carreras, el uso de software específicos (para análisis de datos, simuladores, diseño gráfico entre otros) en algunos casos no pueden ser manipulados desde los celulares. Es oportuno mencionar que, cuando el estudiantado podía asistir a la universidad, muchos resolvían esas carencias a partir de computadoras de uso compartido disponibles en diferentes espacios del establecimiento, por ejemplo: en las bibliotecas, salas de informáticas, en los departamentos o box asignados a las diferentes carreras, así también, quienes podían llevar computadoras a clases las compartían con sus compañeros o cuando podían reunirse en sus hogares las usaban colaborativamente.

Por otro lado, de acuerdo con los datos del MAUTIC 2019, el uso de las tecnologías (celular, computadora e internet) con relación a la edad, muestra variaciones. Se registra el mayor uso de internet en los grupos comprendidos entre los 13 y los 29 años, 90 de cada 100 personas de esas edades lo utilizan. El celular resulta ser la tecnología de uso más extendida para la población joven y adulta. Lo utilizan entre el $94,6 \%$ y el $95,9 \%$ de las personas de 18 a 64 años y el $67,7 \%$ de la población de 65 años y más. Sin embargo, cabe recordar que los datos antes mencionados corresponden al Gran Catamarca y que a la universidad asisten estudiantes de distintos departamentos de la provincia (16 en total) siendo el más cercano el departamento Ambato (a 44 km de distancia) y el más lejano Antofagasta de las Sierras (a 600 $\mathrm{Km}$ ). A su vez el $70 \%$ de la superficie de la provincia es montañoso, lo que también dificulta los procesos de comunicación. 
Es oportuno mencionar que ante la situación de que la mayoría de los estudiantes accede a sus clases mediante el uso de celulares y muchos de ellos lo hacen con servicio prepago, la UNCA a través de la Secretaria de Bienestar y Asuntos Estudiantiles generó el sistema de becas de conectividad denominado Más Conectados, que provee internet de forma gratuita a los estudiantes que tienen problemas de conectividad para que puedan recibir toda la información y el material virtual necesario para continuar con sus clases. La ayuda se otorga según la disponibilidad técnica y necesidades del estudiantado, en tal sentido, a quienes cuentan con PC se les entrega un modem en comodato, y a los que usan el teléfono celular se les habilita un paquete de datos o se les entregará un chip. Desde el inicio de las medidas gubernamentales de ASPO, y al mes julio, ya se otorgaron 600 becas.

Por otro lado, ante el progresivo avance de la COVID-19 el Departamento de Educación a Distancia, dependiente de la Secretaría Académica y de Posgrado de la UNCA, generó el programa denominado Orientaciones técnicas y didácticas para docentes universitarios y preuniversitarios. A través del mencionado programa se desplegaron acciones tendientes a acompañar a los docentes en el proceso de aprendizaje de estrategias de trabajo didáctico en el marco de las orientaciones que provienen del campo de la Educación a Distancia y de los enfoques educativos vinculados al uso pedagógico y didáctico de las TIC. Para el nivel universitario se proyectó el desarrollo de tres acciones concretas: 1) Apertura de aulas virtuales para los docentes de las distintas unidades académicas. 2) La formación intensiva y autogestionada a través de cursos MOOC (Massive Open Online Course) para apoyar el trabajo específico en las aulas virtuales del campus de la UNCA. Los cursos ofrecerán lineamientos teóricos y prácticos básicos en relación con tres dimensiones: la gestión técnica de aulas virtuales, la tutoría académica virtual y los materiales didácticos. 3) Asesoría directa y permanente del equipo de Educación a distancia de la UNCA en las dimensiones básicas del trabajo pedagógico virtual. Así también, desde el mes de marzo, este departamento ofreció a toda la comunidad universitaria, y comunidad educativa en general, instancias de formación reflexiva a través de webinars gratuitos a cargo de especialistas del campo de la Tecnología y Educativa y las Ciencias de la Educación.

En sintonía con las acciones orientadas al acompañamiento y asesoramiento de los docentes se habilitó el sistema de conferencia web de código abierto BigBlueButton (bbb). Esta plataforma admite múltiples comparticiones de audio y video, presentaciones con capacidades ampliadas de pizarra, como puntero, zum y dibujo, chat público y privado, uso compartido de escritorio y al estar alojado en un dominio edu.ar (https://videoconferencia.unca.edu.ar/b) no consume datos móviles de los celulares. Este servicio sin costo se generó por acciones conjuntas entre el Consejo Interuniversitario Nacional (CIN), el Ente Nacional de Comunicaciones (ENACOM), la Secretaría de Políticas Universitarias, el Ministro de Educación de la Nación y las empresas prestadoras de servicios de telefonía e internet. Estas acciones posibilitaron que el ingreso a las plataformas educativas de las 57 universidades nacionales no consuma datos móviles. Asimismo, estas instituciones proyectan el desarrollo de acciones orientadas a fortalecer la infraestructura de conectividad universitaria para dar soporte a la educación superior como objetivo postpandemia.

Las gestiones institucionales ampliaron las posibilidades de continuidad educativa de muchos de los estudiantes, sin embargo, el mayor problema radica en el deficiente servicio de internet que requiere mayores inversiones en la infraestructura telecomunicacional a nivel provincial y nacional. Ante la suspensión de actividades presenciales muchos estudiantes retornaron a sus lugares de residencia permanente y dadas las particularidades de los diferentes departamentos de la Provincia quienes logran acceder a internet, ya sea de forma fija o móvil, tienen problemas con la velocidad del servicio; es muy frecuente escuchar en estos tiempos que internet se corta o es lento, puesto que la rapidez para subir o descargar contenidos será diferente según el tipo de red o medio a través del que se transmiten los datos (inalámbrica, fibra óptica, cables de teléfono o coaxial) y de la cantidad de dispositivos conectados en el hogar. En este sentido a partir de los datos brindados por el Ente Nacional de Comunicaciones, correspondientes al tercer trimestre de 2019, Artopoulos $(2020$, p. 2) señala: "si el hogar dispone de acceso 
a internet, la velocidad y la cantidad de dispositivos en el hogar determinan cuán intensa será la vinculación con el docente. Cuando la velocidad es menor a 20 Mbps se dificultan las actividades de aprendizajes sincrónicos (videoconferencias o foros), solo quedando disponibles las actividades asincrónicas (tarea, lecturas o videos)" (Artopoulos, 2020).

Al momento de escribir este ensayo Argentina superó los 100 días de ASPO, ya en pleno invierno, en la UNCA culmina el primer, y hasta ahora único, cuatrimestre de enseñanza totalmente mediado por tecnologías digitales en carreras de régimen presencial. La continuidad de los procesos pedagógicos requiere de mediaciones pedagógicas y tecnológicas, así como del desarrollo de saberes complejos que permitan transformar las prácticas de la enseñanza en el nivel superior desde la intersección de conocimientos disciplinares, pedagógicos y tecnológicos. Hoy no caben dudas que no alcanza con conocer en profundidad los contenidos de la disciplina y sus posibles formas de representación metodológica, es necesario entender que los conocimientos son provisorios, que el uso de las tecnologías puede contribuir u obstaculizar el logro de finalidades formativas, que el desarrollo tecnológico cambió los modos de construir el conocimiento y que estos procesos no pueden quedar afuera de las prácticas de la enseñanza.

\section{Y AHORA ¿CÓMO ENSEÑAMOS? LAS CONSTRUCCIONES METODOLÓGICAS COMO OPORTUNIDAD PARA RECREAR LAS PRÁCTICAS DE LA ENSEÑANZA Y DESARROLLAR NUEVAS HABILIDADES}

En un mundo donde las tecnologías digitales son ampliamente utilizadas, no puede soslayarse la influencia o ausencia de los procesos de mediación tecnológica en la educación superior. Es necesario que estudiantes y docentes se apropien de competencias tecnológicas (Ferrés y Piscitelli, 2012) que les permitan comprender los nuevos lenguajes comunicativos, intervenir y participar en los campos respectivos de desempeño profesional desde una perspectiva ética, tecnológica y disciplinar.

Durante el trayecto de formación profesional los estudiantes poseen y usan sus saberes, conocimientos, habilidades, aptitudes para seguir aprendiendo y desarrollar nuevas capacidades, estos procesos son mediados por los docentes y diferentes dispositivos que contribuyen a la apropiación de los contenidos. En este sentido, se considera que analizar las formas de comunicación de los conocimientos y cómo los estudiantes se apropian de los mismos en entornos y ámbitos particulares permitirá ampliar las potencialidades de los estudiantes y reinventar (Maggio, 2018) las practicas de la enseñanza del nivel superior.

Se recupera como referente principal para estudiar los procesos metodológicos en las prácticas de la enseñanza universitaria los estudios de Gloria Edelstein (1996, 2002, 2005, 2007, 2011) quién definió la dimensión metodológica del proceso didáctico en términos de construcción metodológica. Este enfoque supera la concepción mecanicista del profesor como aplicador de técnicas y métodos didácticos genéricos. La construcción metodológica da cuenta de la articulación forma-contenido en la construcción didáctica, esta última refiere a "la recontextualización, que realizan maestros y profesores, de los diseños curriculares que operan como regulaciones más generales de sus prácticas" (Edelstein, 2011: 181). La idea de construcción didáctica concibe la enseñanza como un proceso complejo de gestación de propuestas de intervención que ponen en juego la relación dialéctica contenido-método. La construcción metodológica remite a un mayor nivel de concreción cuando la recontextualización curricular alude a contextos y sujetos concretos "desafiando resoluciones creativas de la relación dialéctica forma-contenido para la enseñanza en ámbitos singulares" (Edelstein, 2011: 181).

La construcción metodológica da cuenta por parte del profesor de un acto singularmente creativo a partir de la cual concreta la articulación entre las lógicas del contenido (lo epistemológico objetivo), lo relativo 
a la producción ya existente respecto del contenido (su historia del desarrollo y sus principales debates); las lógicas de los sujetos (lo epistemológico subjetivo), que remiten a las posibilidades cognitivas, motoras, afectivas, sociales de apropiación de los contenidos; y, por último, aquellas que aluden a los ámbitos y contextos en las que las dos lógicas mencionadas se entrecruzan. Construcción casuística, singular, por tanto relativa, que se genera - por parte de maestros y profesores- a diferentes escalas (programa, unidad, clase) por la articulación de macro y micro decisiones respecto de las propuestas de intervención a los fines de la enseñanza (Edelstein, 2011: 177-178)

En este sentido es fundamental conocer la diversidad de enfoques y modos de construcción del conocimiento propio de cada campo y área disciplinar que posibiliten procesos cognitivos y metacognitivos orientados a la apropiación de los conocimientos por parte de los estudiantes. Para lo cual "es imprescindible diseñar actividades de distinto tipo con el objeto de generar la construcción de conocimientos por parte del alumno" (Edelstein, 2011:179). El punto clave que ofrece la noción de construcción metodológica radica en la posibilidad de crear que tiene el docente, creación que se configura y define pensando en los otros.

Esta perspectiva otorga a quien enseña una dimensión diferente. Deja de ser actor que se mueve en escenarios prefigurados para constituirse en sujeto creador; sujeto que imagina, produce diseños alternativos que, en lo esencial, den lugar a la reconstrucción del objeto de enseñanza por parte del sujeto que aprende. Diseños que le otorgan al alumno la posibilidad de recrear, de resignificar lo que es transmitido; que lo coloque no en situación de posesión-reproducción, sino de sujeto en búsqueda de posibles recorridos, nuevos atajos más allá de cualquier camino preestablecido (Edelstein, 2011: 179).

Como se expresó en párrafos anteriores, la pregunta por el sujeto de la formación y con ella sentido pedagógico del trayecto que realiza el estudiantado, era una tarea pendiente en relación con la multiplicidad de tareas que se despliegan en el ámbito universitario. El creciente y continuo desarrollo tecnológico impacta los procesos de construcción de las subjetividades entendidas como "modos de hacer en el mundo, con el mundo, como serie de operaciones que realiza el sujeto para habitar el mundo" (Corea, 2005). Estos nuevos modos de ser y estar desestabilizan las prácticas pedagógicas tradicionales, por lo que no puede soslayarse la influencia o ausencia de los procesos de mediación tecnológica en la educación superior como nivel abocado a la formación de profesionales que se desempeñarán en un mundo cada vez más tecnologizado (Diaz, 2017; Diaz y Yuni, 2017; Diaz y Sánches Escalante, 2018).

La máxima expresión de las alteraciones que provocan las tendencias tecnológicas y culturales contemporáneas y la necesidad de que los sujetos pedagógicos desarrollen habilidades digitales es el salto a la virtualidad provocado por la pandemia. Las construcciones metodológicas expresan y materializan decisiones de los docentes que habilitan u obturan la posibilidad de creación de nuevos conocimientos que entramen los saberes disciplinares, tecnológicos, humanísticos y sociales que se pretende que todo profesional aprenda. Los eventos que alteraron los actuales escenarios y entornos educativos llevan a profundizar y ampliar el sentido que se le otorga a la categoría construcción metodológica en tanto hoy, y mientras no se reanuden la modalidad de enseñanza presencial, esas creaciones solo son posibles con mediaciones tecnológicas.

Actualmente las plataformas de los medios sociales como YouTube ${ }^{\oplus}$, Facebook ${ }^{\circledR}$, Twitter $^{\circledR}$, Instagram ${ }^{\circledast}$, WhatsApp ${ }^{\oplus}$, y algunas de las popularizadas por la pandemia como Zoom $^{\oplus}$, Google Meet ${ }^{\oplus}$, Jitsi ${ }^{\oplus}$ alteraron la naturaleza de la comunicación pública y privada y constituyen "un ecosistema de medios conectivos que nutre y a su vez se nutre de normas sociales y culturales que pasan por un proceso de evolución simultáneo dentro del mundo cotidiano" (Van Dijck, 2016 p. 43). Estos ecosistemas tecnológicos "se caracterizan por la integración de componentes software heterogéneos para proporcionar un conjunto de funcionalidades que cada componente por separado no ofrece, así como mejorar la experiencia de 
los usuarios, considerándoles un elemento más dentro del ecosistema, característica fundamental y diferencial en esta aproximación" (De Pablos y otros, 2019: 66).

La posibilidad de usar la multiplicidad de medios disponibles y el acceso a Internet, aun con todas sus dificultades, permite dar cierta continuidad al proceso educativo en el sentido de que a través de estas herramientas es posible representar, procesar, transmitir y compartir información. No obstante, desde una perspectiva socio constructivista del aprendizaje, Coll $(2004$, p. 9) señala:

(...) ni información es sinónimo de conocimiento ni la recepción o el acceso a la información garantiza el aprendizaje. La información se convierte en conocimiento y el acceso a la información da lugar al aprendizaje cuando actuamos sobre ella, la procesamos, la organizamos, nos la apropiamos, la utilizamos y la confrontamos con otros; en suma, cuando somos capaces de darle significado y sentido.

Desde esta perspectiva, las habilidades que posibilitan la transformación de la información en conocimiento son resultado de complejos procesos interactivos y comunicativos. Para comprender los procesos de mediación tecnológica que se despliegan en las construcciones metodológicas universitarias se recuperan los aportes de Coll, Mauri y Onrubia (2008) quienes destacan el potencial de las TIC como instrumentos psicológicos mediadores de los procesos intra e inter mentales implicados en los procesos de enseñanza y aprendizaje. Estos investigadores afirman que la novedad que introducen las TIC y las tecnologías digitales radica en que permiten crear entornos que integran los sistemas semióticos conocidos y amplían la capacidad humana para (re)pensar, recrear y compartir gran cantidad de información de forma instantánea y con menos limitaciones de espacio y de tiempo.

Actualmente se amplió de manera impensada las posibilidades de comunicación de los ciudadanos, no obstante, junto a esas oportunidades, los medios logran niveles de acumulación y concentración de información a los que nunca habían llegado. Los ecosistemas tecnológicos generan nuevas demandas formativas al campo educativo y una de ellas es la necesidad de que estudiantes y profesores se apropien de competencias mediáticas como posibilidad de potenciar la excelencia personal. "La competencia mediática ha de contribuir a desarrollar la autonomía personal de los ciudadanos y ciudadanas, así como su compromiso social y cultural" (Ferres y Piscitelli, 2012, p. 76). Para desarrollar este tipo de competencias no se puede obviar las reales posibilidades de uso, posesión de dispositivos tecnológicos y acceso a la conectividad puesto que la carencia de recursos tecnológicos provoca una posesión diferencial de habilidades. El concepto brecha digital refiere a que el uso de computadoras e internet genera beneficios significativos mientras que el no uso de estos tiene consecuencias negativas. Razón por la cual, se demanda a las instituciones educativas la enseñanza y aprendizaje de habilidades digitales que permitan reducir tales brechas.

Las investigaciones de Van Deursen, Van Dijk y Peters (2017) brindan una tipología de habilidades digitales susceptibles de ser desarrolladas en relación con los aspectos técnicos del medio y el contenido. Distinguen cuatro tipos de habilidades digitales: habilidades operacionales y formales (relacionadas con el medio) y habilidades de búsqueda de información y estratégicas (relacionadas con el contenido). Las habilidades operacionales remiten al uso básico de internet (por ejemplo, utilizar los motores de búsqueda introduciendo términos específicos en los campos apropiados). Las habilidades formales se vinculan a la estructura hipermedia sobre la que se construye internet (tales como navegar y orientarse en entornos digitales). Con relación al contenido, se incluyen las habilidades de búsqueda de información (por ejemplo, encontrar noticias o datos definiendo opciones o preguntas de búsqueda) mientras que las habilidades estratégicas son aquellas que permiten utilizar internet como medio para alcanzar objetivos particulares, así como para mejorar la posición social de individuo (usar la web en relación con cierto objetivo específico). Estas habilidades son individuales y necesarias para que la población sea capaz 
de desenvolverse en entornos digitales cada vez más complejos y al tener en cuenta tanto los aspectos técnicos del medio como el contenido se evita un punto de vista tecnológicamente determinista.

En relación con lo anterior, si analizamos los procesos de adquisición de habilidades digitales según la edad y nivel educativo de los sujetos, son relevantes los estudios que señalan que las personas adultas, que cursan o culminaron estudios superiores, desarrollan mayores habilidades relacionadas con el contenido.

(...) si bien, cuanto menor es la edad, mayores son las competencias relacionadas con el manejo operacional y formal de los medios digitales, las personas adultas despliegan mayores habilidades relacionadas con el contenido buscado, procesado y utilizado en Internet al tiempo que se destaca que, por encima de la edad, el factor con mayor poder explicativo de las capacidades diferenciales resulta ser el nivel educativo (Van Deursen, Dijk y Peters, 2017, p. 149 en Benítez Larghi y Ugarte, 2019, p. 6).

Si se concibe el conocimiento en el marco de las condiciones materiales de producción, circulación y apropiación, las habilidades digitales deben comprenderse "(...) como el resultado de las interacciones entre sujetos y objetos construidas en el marco de determinadas relaciones de poder moldeadas dentro del modo de producción capitalista contemporáneo". (Benítez Larghi, 2020, p. 150)

Respecto al desarrollo de habilidades digitales en escenarios educativos interpelados y entramados en plataformas de medios conectivos, los estudios de Casillas Alvarado, Ramírez Martinell y Ortiz Méndez (2014), basándose en la categoría capital cultural propuesta por Bourdieu (1987), advierten que en los escenarios educativos mediados por TIC comienza a operar una nueva especie de capital: el capital tecnológico "el cuál (...) funciona como recurso que se invierte y se ejerce en determinadas relaciones sociales, como cualquier capital está desigualmente distribuido y su posesión otorga ventaja en la competencia en el campo respectivo" (p. 26). Su posesión es un atributo que diferencia a los individuos y les permite competir de mejor manera en muy diversos campos y espacios sociales. En relación con ello se plantea la relevancia del aprendizaje de habilidades digitales que potencien la posesión de un capital tecnológico que amplíe las posibilidades de intervención y participación social de todos los ciudadanos.

Los procesos de mediación tecnológica contemplan tanto los aspectos técnico-instrumentales que influyen en el diseño tecno pedagógico (Coll, Mauri y Onrubia: 2008) como las dimensiones simbólicas y subjetivas ancladas en acciones particulares y colectivas. Las construcciones metodológicas mediadas con tecnologías digitales en tiempos de aislamiento social, aun con todos los desafíos que conllevan, son una oportunidad para alterar los formatos curriculares, los modelos didácticos, las dinámicas del aula y las formas de organización universitaria. Los ecosistemas tecnológicos que configuran los escenarios contemporáneos y la necesidad de desarrollar habilidades digitales dan cuenta de que las tecnologías no son solo instrumentos sino "formas de estar en el mundo" (Baricco, 2019). Estas formas de habitar el mundo se entraman a un conjunto de fenómenos y avances tecnológicos como el Big Data, la inteligencia artificial, dataficación, robótica que cambiaron los modos de vincularnos con los otros e interpelan las aulas universitarias como espacio de construcción colectiva del conocimiento.

El desarrollo de nuevos saberes y habilidades durante la formación superior implica el replanteo de los tradicionales modos de enseñar en la universidad. La inclusión genuina de las tecnologías (Maggio, 2012) a las aulas universitarias está determinada por conocimientos del orden cultural, epistémico y didáctico que les da sentido y aprovecha estas nuevas tecnologías para incorporarlas al sistema productivo y al beneficio social. Hoy en día, la antigua idea de compartir códigos y respetar leyes universales que sostengan la trasmisión de conocimientos de arriba hacia abajo -característico del dispositivo pedagógico moderno- dejó de ser un mito para convertirse en un anacronismo (Sibila, 2016). 
Desde el inicio de este ensayo se expresó que la pandemia puso en jaque los modos de construir las prácticas de la enseñanza en el nivel superior, reflotando viejos e instalando nuevos problemas que evidenciaron con más fuerza los sesgos de las tradiciones pedagógicas academicistas que aun hoy conforman el ámbito universitario. Sin embargo, por la complejidad de la que somos parte, la situación de emergencia sanitaria se constituye en un desafío que provoca la renovación de las clases y aulas universitarias.

Sin dejar de cuestionar el solucionismo tecnológico, esta situación de emergencia global debiera ser el punto de partida definitivo para disipar fronteras arquitectónicas, consolidar procesos de innovación en las formas de enseñanza remota y naturalizar la cultura digital en la educación superior (Pardo Kuklinsky y Cobo, 2020, pp. 13-14).

Replantear el sentido de la formación era una actividad pendiente de los claustros universitarios. Sin embargo, la incertidumbre en que vivimos afianza la idea de la formación como "la dinámica de un desarrollo personal" (Ferry, 1997, p.54) que para su concreción necesita espacios y tiempos de formación que faciliten la reflexión a través de otros mediadores. No se recibe formación, sino que se busca, en un proceso activo que requiere mediaciones pedagógicas y tecnológicas que posibiliten diálogos genuinos entre estudiantes, formadores, organizaciones e instituciones sociales, que a veces se consideran el afuera de la universidad, porque afortunadamente el conocimiento dejó de ser un objeto dado para ser una construcción colectiva, colaborativa y en constante renovación.

Un análisis de la enseñanza que ya no se considera patrimonio exclusivo de los docentes, establece nuevos modelos relacionales y participativos en las prácticas educativas mediante redes de conocimiento. Durante las últimas décadas, hemos visto cómo se ha ido cuestionando el conocimiento inmutable de las ciencias y se abren otras concepciones en las cuales la interpretación, la comprensión de la realidad, la incertidumbre y la complejidad tienen un papel importante en la realidad científica y social. (Lion, 2020, p. 19)

En el marco de las actividades investigativas desarrolladas desde enero del año 2019 en el Proyecto de investigación denominado Inclusión tecnológica y construcciones metodológicas en las prácticas de enseñanza universitaria. Aportes para reconstruir la Didáctica Universitaria, aprobado por la Secretaria de Investigación y Posgrado de la UNCA, se pretendía sistematizar construcciones metodológicas innovadoras que incluyan, en prácticas de la enseñanza universitarias de régimen presencial, el uso de las tecnologías digitales y demanden la puesta en juego de competencias mediáticas. Sin embargo, en virtud de los acontecimientos y reflexiones teóricas expresadas en este ensayo, actualmente se trabaja en el proceso de revisión de los posicionamiento teóricos y metodológicos asumidos antes de la pandemia, proceso que dio lugar a nuevas preguntas: ¿cuáles son las plataformas más utilizadas para la enseñanza en el ámbito universitario de la provincia de Catamarca?, y en ellas, ¿cuáles son las funcionalidades, que, en su combinación, mejoran la experiencia educativa y el desarrollo de habilidades digitales de docentes y estudiantes?, ¿cómo conocer las clases universitarias cuando hoy son posibles por mediaciones digitales y se despliegan entre el escenario doméstico y los ecosistemas de medios conectivos? Así también, pensar la enseñanza como práctica social, humana y política requiere interrogarla en clave de inclusión pedagógica ¿A quiénes excluyen estas formas de mediación tecnológica de los conocimientos? Como lo señala Kap (2020, p. 8) "es cierto que las plataformas y los recursos tecnológicos lograron establecer lazos comunicativos y pedagógicos potentes, también lo es que en algunos casos los conflictos se agravaron. La falta de posibilidad de conectarse de algunos estudiantes los deja aún más excluidos". 
Construir propuestas metodológicas mediadas con tecnologías digitales requiere saber de qué modo la tecnología ayuda a resolver problemas vinculados a la enseñanza y el aprendizaje, si puede o es modificado el contenido de la enseñanza al momento de incluir una herramienta tecnológica, qué capacidades y habilidades pueden ampliar, mejorar o descubrir docentes y estudiantes mediante la tecnología, quiénes se benefician y se perjudican con esas mediaciones. Los actuales escenarios sociales y educativos son confusos y difíciles, sin embargo, también son una oportunidad para reconstruir la didáctica del nivel superior mediante la investigación rigurosa de las nuevas o renovadas formas de enseñar en la universidad durante y después de la pandemia.

Aún no se sabe cuándo, ni cómo, será el retorno a las universidades. Pero, sí se reconoce la necesidad de anticipar posibles alternativas de intervención pedagógica y didáctica que contemplen las reales posibilidades de acceso a la educación, teniendo en cuenta las lógicas del conocimiento y los modos de apropiación de estos por parte de los sujetos en formación, así como los diversos medios tecnológicos disponibles y su desigual potencial como herramienta educativa. A lo que se suma, en tiempos de pandemia, los eventos que pudieran ocurrir vinculados a los efectos económicos, sociales y políticos de un aislamiento social preventivo y obligatorio que en Argentina inició hace más de 100 días.

\section{REFERENCIAS}

Artopoulos, A. (2020). ¿Cuantos estudiantes tienen acceso a Internet en su hogar en Argentina? Buenos Aires: Observatorio Argentinos por la Educación. Recuperado de https://cms.argentinosporlaeducacion.org/media/reports/ArgxEdu_Conectividad_Coronavirus_.pdf

Baricco, A. (2019) The Game. Anagrama

Basabe, L. y Cols, E. (2007) La enseñanza. En A. Camilloni y otras El saber didáctico (pp. 125-158) Buenos Aires. Argentina: Paidós.

Benítez Larghi, S. (2020). La construcción de habilidades digitales estudiantiles en torno al Programa Conectar Igualdad. Ciencia, Docencia y Tecnología, 31 (60 may-oct), 131-154. https://doi. org/10.33255/3160/581

Benítez Larghi, S., y Ugarte, D. (2019). Más allá de nativos e inmigrantes. Trayectorias generacionales de apropiación de internet en dos conglomerados urbanos de Sudamérica. Question/Cuestión, 1(64). https://doi.org/10.24215/16696581e209

Bolivar A. y Bolivar Ruso, R. (2011) La didáctica en el núcleo del mejoramiento de los aprendizajes. Entre la agenda clásica y actual de la Didáctica. Revista Perspectiva Educacional, 50 (2), 3-26

Bourdieu, P. (1987). Los tres estados del capital cultural. Revista sociológica 2 (5). Recuperado de http:// www.sociologicamexico.azc.uam.mx/index.php/Sociologica/article/view/1043/1015

Camilloni A. (1996) De herencias deudas y legados. Una introducción a las corrientes actuales de la didáctica. En A. Camilloni y Otras. Corrientes didácticas contemporáneas (pp. 17-40) Buenos Aires. Editorial Paidós

Camilloni A. (2007) Didáctica general y didácticas específicas en A. Camilloni y otras El saber didáctico (pp. 23-39). Buenos Aires. Argentina. Paidós.

Casillas Alvarado, Ramírez Martinell y Ortiz Méndez (2014) El capital tecnológico una nueva especie de capital cultural. Una propuesta para su medición en Ramírez Martinell, A. y Casillas Alvarado M. Háblame de TIC. Tecnología digital en la educación superior (pp. 23-38) Editorial Brujas. Argentina.

Coll, C. (2004). Psicología de la educación y prácticas educativas medidas por las tecnologías de la información y la comunicación. Una mirada constructivista. Revista electrónica Sinéctica, 25, 1-24. 
Coll, C., Mauri, T., \& Onrubia, J. (2008). Análisis de los usos reales de las TIC en contextos educativos formales: una aproximación sociocultural. Revista Electrónica de Investigación Educativa, 10(1). Recuperado de http://redie.uabc.mx/vol10no1/contenido-coll2.html

Corea, C. (2004) Pedagogía y Comunicación en la era del aburrimiento en C. Corea, e I. Lewkowicz Pedagogía del Aburrido. Escuelas destituidas, familias perplejas. (pp. 41-70) Buenos Aires: Ed. Paidós

Davini, M. C. (2008) Métodos de enseñanza. Didáctica general para maestros y profesores. Buenos Aires: Santillana.

De Pablos, J.M., Colás, M.P., López Gracia, A. y García-Lázaro, I. (2019). Uses of digital platforms in Higher Education from the perspectives of the educational research. REDU. Revista de Docencia Universitaria, 17 (1), 59-72. https://doi.org/10.4995/redu.2019.11177

Presidencia de la Nación Argentina. (2020). Decreto n²97 de 2020 [Poder Ejecutivo] por el cual se establece la medida de Aislamiento Social Preventivo y Obligatorio. 19 de marzo 2020. Presidencia de la Nación Argentina. Recuperado de https://www.boletinoficial.gob.ar/detalleAviso/ primera/227042/20200320

Diaz A. G. (2017) Reconfiguración pedagógica didáctica de la formación docente: el uso de aulas virtuales. En M. Insaurralde y otras (comp.) (2017) Tender puentes: para enseñar y aprender en la educación superior (886-894). Argentina: Editorial Universidad Nacional de Luján.

Diaz A. G. y Sánchez Escalante M. C. (2018) Capitulo 8: Usar las tecnologías en la escuela secundaria tensiones didácticas en los procesos de inclusión tecnológica en J. Yuni (director) Inclusión/es en la escuela secundaria. Itinerarios de lo posible. (pp. 159-186) Córdoba Encuentro Grupo Editor. Catamarca CITCA.

Díaz, A. G., y Yuni, J. A. (2017). Desplazamientos y tensiones en las gramáticas formativas de futuros profesores: análisis de una experiencia didáctica en la Universidad. Revista Fuentes, 19(2), 111-124. doi: http://dx.doi.org/10.12795/revistafuentes.2017.19.2.08

Edelstein, G. (1996) Un capítulo pendiente: el método en el debate didáctico contemporáneo en A. Camilloni y otras Corrientes didácticas contemporáneas. (pp. 75-90) Argentina. Paidós.

Edelstein, G. (2002). Problematizar las prácticas de la enseñanza. Perspectiva, 20(2), 467-482. doi:https:// doi.org/10.5007/\%x

Edelstein, G. (2011) Formar y formarse en la enseñanza. Bs. As. Paidós

Edelstein, G., Salit, C., Gabbarini, P., Domjan, G. y Sívori Fernández, L. (2007) Los indicios acerca de la construcción de conceptos sobre lo metodológico en la enseñanza. Cuadernos de educación. V (5), 141-157 Córdoba. Imprenta facultad de Filosofía y Humanidades.

Ferrés, J., y Pisticelli, A. (2012). La competencia mediática: propuesta articulada de dimensiones e indicadores [Media Competence. Articulated Propos a lof Dimensions and Indicators]. Comunicar, 19(38), 75-82. doi: 10.3916/C38-2012-02-08.

Ferry G. (1997) Pedagogía de la formación. Colección formación de formadores. Bs. As. Ediciones Novedades Educativas.

Instituto Nacional de Estadística y Censos (2019) Acceso y uso de tecnologías de la información y la comunicación. (Informes Técnicos. Vol. 4, no 1 ) Encuesta permanente de Hogares Cuarto trimestre de 2019 ISSN 2545-689X recuperado de https://www.indec.gob.ar/uploads/informesdeprensa/ mautic_05_20a36AF16B31.pdf consultado 11/06/2020

Kap, M. (2020) Mutaciones de la Enseñanza y el Aprendizaje en tiempos de Pandemia. Revista Enlace Universitario $\mathrm{n}^{\circ} 34$ - ISSN 1850-2490/. Disponible en https://www3.mdp.edu.ar/attachments/article/68/Enlace34.pdf

Lion C. (comp.) (2020). Aprendizaje y tecnologías. Habilidades del presente, proyecciones de futuro https:// digital.noveduc.com/reader/aprendizaje-y-tecnologias?location=19 
Litwin, E. (1996) El campo de la didáctica: búsqueda de una nueva agenda en A. Camilloni y Otras Corrientes didácticas contemporáneas. (pp. 41-76) Argentina. Paidós.

Maggio, M. (2 de noviembre de 2016). Programa la UBA para el siglo XXI. Las clases re-concebidas en ambientes de alta disposición tecnológica. Recuperado de https://www.youtube.com/ watch?v=U3ELBJUiMI4

Maggio, M. (2012) Enriquecer la enseñanza. Los ambientes con alta disposición tecnológica como oportunidad. Buenos Aires: Paidós

Maggio, M. (2018) Reinventar la clase en la universidad. Buenos Aires: Paidós

Meléndez, C. E., Díaz, A. G. y Yuni, J.A. (2017). Políticas de inclusión digital en la formación docente. Revista del Cisen Tramas/Maepova, 5 (2), 75-91.

Morin E. (2000) [1994] Introducción al Pensamiento Complejo. $3^{\circ}$ reimpresión de la $1^{\circ}$ edición. España, Editorial Gedisa.

Pardo Kuklinski, H. y Cobo, C. (2020). Expandir la universidad más allá de la enseñanza remota de emergencia Ideas hacia un modelo híbrido post-pandemia. Outliers School. Barcelona.

Pruzzo V. (comp.) (2015) Didáctica general. Investigación empírica y discusiones teóricas. Argentina. Instituto Superior de estudios psicopedagógicos y sociales

Resolución 104 de 2020 [Ministerio de Educación Nacional] Recomendaciones de adecuación de la actividad académica del nivel superior en el marco de la emergencia COVID-19. 14 de marzo de 2020. Disponible en https://www.argentina.gob.ar/sites/default/files/rs-2020-16985574-apn-me.pdf

Resolución Rectoral n¹02 de 2020 [Universidad Nacional de Catamarca]. Por la cual se faculta a las autoridades de las Unidades Académicas a la reprogramación de las clases presenciales y la suspensión transitoria de todas las actividades académicas. 16 de marzo de 2020.

Sibila, P. (2016) Entre redes y paredes: enseñar y aprender en la cultura digital. En M. T. Lugo (Coord.), Entornos digitales y políticas educativas: dilemas y certezas (pp. 201-224). Buenos Aires: Instituto Internacional de Planeamiento de la Educación IIPE-Unesco.

Souto, M. (2009) Complejidad y formación docente. En J. Yuni (comp.) La formación docente complejidades y ausencias (pp. 13-26). Córdoba: Brujas

Van Deursen, A. J. A. M., Van Dijk, J. A. G. M., y Peters, O. (2017) Habilidades digitales relacionadas con el medio y el contenido: la importancia del nivel educativo: Medium and content related Digital Skills: the importance of education level of attainment. Panorama Social, 25, 137-152. [10].

Van Dijck J. (2016) La cultura de la conectividad. Una historia crítica de las redes sociales. Siglo XXI editores. Argentina 